\title{
CALIBRATED MULTI-TEMPORAL EDGE IMAGES FOR CITY INFRASTRUCTURE GROWTH ASSESSMENT AND PREDICTION
}

\author{
R. Al-Ruzouq * , A. Shanableh, Z. Boharoon, M. Khalil ${ }^{1}$ \\ Dept. of Civil and Environmental Engineering, University of Sharjah, UAE - ralruzouq@ sharjah.ac.ae
}

Commission VI, WG VI/4

KEY WORDS: Urbanization, Edge images, Urban, Land Use, Infrastructure Growth, Multi-Temporal Landsat

\begin{abstract}
:
Urban Growth or urbanization can be defined as the gradual process of city's population growth and infrastructure development. It is typically demonstrated by the expansion of a city's infrastructure, mainly development of its roads and buildings. Uncontrolled urban Growth in cities has been responsible for several problems that include living environment, drinking water, noise and air pollution, waste management, traffic congestion and hydraulic processes. Accurate identification of urban growth is of great importance for urban planning and water/land management. Recent advances in satellite imagery, in terms of improved spatial and temporal resolutions, allows for efficient identification of change patterns and the prediction of built-up areas. In this study, two approaches were adapted to quantify and assess the pattern of urbanization, in Ajman City at UAE, during the last three decades. The first approach relies on image processing techniques and multi-temporal Landsat satellite images with ground resolution varying between 15 to 60 meters. In this approach, the derived edge images (roads and buildings) were used as the basis of change detection. The second approach relies on digitizing features from high-resolution images captured at different years. The latest approach was adopted, as a reference and ground truth, to calibrate extracted edges from Landsat images. It has been found that urbanized area almost increased by 12 folds during the period 1975-2015 where the growth of buildings and roads were almost parallel until 2005 when the roads spatial expansion witnessed a steep increase due to the vertical expansion of the City. Extracted Edges features, were successfully used for change detection and quantification in term of buildings and roads.
\end{abstract}

\section{INTRODUCTION}

Urban growth can refer to the expansion of a metropolitan or suburban area into the surrounding environment. This expansion in cities can be clearly observed in terms of increased buildings and roads that essentially support population growth and their associated social, economic and environmental needs. The infrastructure improvement is vital to promote economic prosperity and urban development, as they provide access to business activities, employment, education, and recreational opportunities (Al-Ruzouq et al., 2017; Verma et al., 2009).

Although urbanization (i.e., increase in the number of people and accompanying growth in infrastructure, both horizontally and vertically) is desirable for human development, uncontrolled urbanization in cities and developed countries has been responsible for many problems resulting in inadequate living environment, scarcity in drinking water, noise and air pollution and traffic congestion (Liu et al., 2015). For example, the increase of impervious surface area and degradation of natural vegetation during urbanization cause changes in hydraulic processes, loss of existing drainage capacity and flooding in urban areas (Li et al., 2016; Suriya and Mudgal, 2012; Zope et al., 2016). It is therefore of high interest for government officials, planners, decision-makers, and researchers to obtain quantifiable records for the spatial and temporal growth of any city, mainly in terms of transportation infrastructure and buildings (Al-Ruzouq et al., 2017).

Latest knowledge in remote sensing and satellite-based systems, facilitate collecting physical and spatial data on a repetitive basis, as well as simplify integration with Geographic
Information System (GIS) to analyze the data spatially and generate various earth models for optimizing the urban planning process. These information systems also provide connection of geospatial data with socio-economic records, and thereby presenting an essential conceptual view for a meaningful and effective planning process (Verma et al., 2009). Remote sensing detection techniques are implemented to create the necessary data sets to facilitate training of urban growth prediction models. These models may build using historical information extracted from archive images to produce a more accurate change map and incorporated in future monitoring tasks (Jin and Mountrakis, 2013).

Various strategies have been considered for urban growth monitoring through applying change detection procedure along multi-temporal satellite imagery, which is the process of identifying differences in the state of an object or phenomenon by observing it at different times (Singh, 1989). This process includes the ability to quantify changes using multi-resolution, multi-spectral, and/or multi-source imagery captured at different times. Traditional change-detection studies are based on a visual/manual comparison of temporal datasets, such as satellite scenes, aerial images, or maps. However, the vast number of imagery and emergence of new satellite sensors (for example, QuickBird, GeoEye and WorldView) that are able to provide very high spatial resolution images (VHSR) is creating high opportunities for development of automatic, reliable, and fast change-detection techniques that essentially to reducing the high cost associated with spatial data updating and urban growth monitoring (Al-Ruzouq et al., 2017).

\footnotetext{
Corresponding author
} 
The choice of the most appropriate satellite image to be used for change detection depends on many factors; cost, availability and physical characteristics of the images such as spatial, spectral and temporal resolution. VHSR relatively result in more accurate identification of features (roads and buildings) that usually appears in cities and used to quantify the urban growth development (Bouziani et al., 2010; Jin and Mountrakis, 2013). In spite of all the solutions proposed by previous research, the automatic map updating based on VHSR satellite images remains challenging matter (Bouziani et al., 2010). The first issues refer to the fact that VHSR images have high cost and not frequently existing, moreover historical archive images, which are essential for assessing the development pattern of cities, are not always available. The second major issues refer to the process of automatically extraction features based on image processing, where algorithms, storage, image registration, image resampling appears. As an alternative to the automatic extraction is the manual digitizing of features from VHSR. Digitizing is simply defined as the process of "tracing," in a geographically correct way, information from images/maps, such as buildings, roads, and land characteristics, however, digitizing process required numerous efforts of skilled operators, which can be considered as a challenging process that may not be really convenient for continues updating of urban maps.

On the other hand, images acquired by multispectral (MS) medium-resolution sensors have been wildly as a data source in remote sensing and change detection. For example, Landsat satellite images with $(15-79 \mathrm{~m})$ ground resolution are freely available to download every 16 days. These images are archived for more than 4 decades and can be considered as low storage, fast processing, and time-saving material. It has been broadly used for monitoring urban growth (Al-ruzouq et al., 2011; Aljoufie et al., 2013; Elmahdy and Mohamed, 2016; Liu et al., 2015; Rimal, n.d.; Song et al., 2016; Tanr and Canaz, 2017; Verma et al., 2009; Wang et al., 2017; Yildiz and Doker, 2016). Multispectral information is not always sufficient to provide reliable land cover/land-use discrimination; different classes may not be distinguishable due to similar spectral responses (Jin and Mountrakis, 2013). The variations in image properties (e.g., pixel radiance value, texture, and shape) can be related to changes on the ground at different satellite observation times and other external factors such as variation in atmospheric conditions, sensor conditions, illumination difference, and seasonal effects (Liu et al., 2015).

Instead of working with the original images, after intensity modification, one can use derived edge images as a basis for the change detection procedure. Utilizing the edge images has two advantages. First, derived edges are robust to possible radiometric differences between the registered images (e.g., due to noise and/or different spectral properties). Also, the edges would correspond to interesting features (such as building boundaries, roads, and trails). Therefore, comparing edge images will be useful in outlining the amount of urbanization activities, which is one of the most important objectives of this study (Al-ruzouq et al., 2011). Digitized features from VHSR can be used to refine and calibrate edges detected from Landsat images. Mathematical Model can be developed to the depicted relation between extracted edges and digitizing features to assess future urban growth.

This work suggests a methodology to quantify and analyze and urbanization growth in Ajman City/UAE. Over the past four decades, Ajman city has witnessed massive growth in its population, urban area, and infrastructure. For instance, the population of Ajman increased almost 15 fold between 1975 and 2015 as indicated by demographics of UAE. Multi- temporal satellite imagery based on image processing techniques (edge extraction for features such as roads and buildings) were used to get quantitative measurements for urban growth. Prior to change-detection implementation, image-toimage registration was conducted to ensure the accuracy of the change-detection process. The main objectives of this work can be summarized as follows:

1. Quantifying and assessing the pattern of urbanization in Ajman City based on multi-temporal VHSR satellite images.

2. Utilizing edges (buildings and roads) shown in the multi-temporal Landsat satellite images as the base of reliable change detection.

3. Validating the extracted features (Landsat) and change detection using digitized features from VHSR multi-temporal images.

4. Predicting future built-up areas (Roads and buildings) based on extracted edges from Landsat moderate resolution satellite images and archived digitized features as ground truth.

The next section discusses the description of the study area; followed by sections discussing the proposed methodology applied to quantify the urban growth of Ajman City. After that, the utilized data and results of applying the proposed methodology; and the paper concludes with a summary of major findings and recommendations for future research.

\section{STUDY AREA}

The proposed methodology has been applied to Ajman City in the United Arab Emirates, a city located in the northern part of the UAE along the northern coast of the Arabian Gulf of the Arabian Peninsula (central coordinates of $25^{\circ} 25^{\prime} \mathrm{N} 5530^{\circ} \mathrm{E}$ ). It has a dry semiarid climate with a daily high temperature of $24-42^{\circ} \mathrm{C}$ and a daily mean temperature of $18-34^{\circ} \mathrm{C}$. Ajman City, and the UAE in general has faced massive urbanization and expansion since the 1970s. Figure 1(a) and (b) show the UAE map with respect to its neighboring countries, and the study area of Ajman City, respectively. The study area extended over $\sim 143.00 \mathrm{Km} 2$.

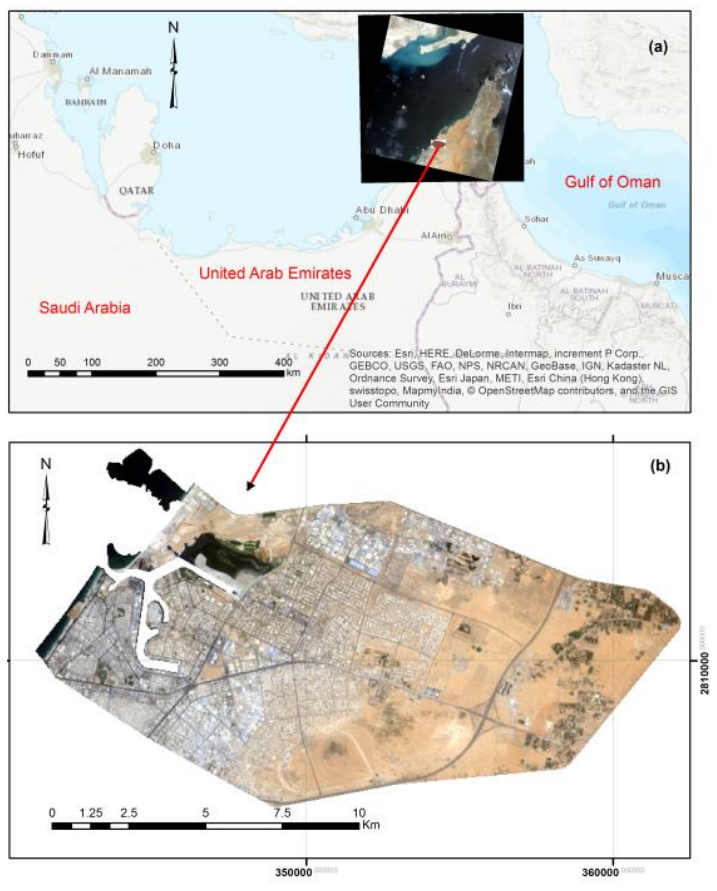

Figure 1. The Study Area: (a) Location Map; (b) Ajman City. 
As shown in Figure 2, the framework of proposed methodology relies on two sets of Satellite image with different radiometric and geometric resolutions that been captured for different years; Landsat and VHSR Satellite Images. Landsat satellite images with the highest available spatial resolution were selected based on their availability while maintaining approximately a 10 -year analysis period. Similarly, QuickBird, GeoEye_1 and WorldView_2 Satellite multi-temporal images were used to represent ground truth; therefore, time synchronization with selected Landsat images was considered, in addition to selecting the highest possible spatial resolution image (i.e., $<1$ meters). It has to be mentioned digitized images from registered multitemporal VHRS) were prepared and used as a reference for all Landsat images. Table 1 lists each image used including the source, the year of capturing the image, and its spatial resolution in meters.

In summary, seven Landsat and four VHSR images were captured and geo-referenced to cover the spatiotemporal aspects of the study area. Figure 3(a) shows all the Landsat images for the study area for the years 1975 to 2015; while Figure 4 (a) shows a sample of WorldView Satellite images with 0.46-meter ground resolution and a close up sample in Figure 4 (b). Such high resolution enabled accurate digitizing of the features, namely roads and buildings of the city.

\begin{tabular}{|c|l|c|}
\hline Year & Satellite Image & Resolution \\
\hline \multirow{3}{*}{1975} & Landsat (MSS) & $\mathrm{m}$ \\
1985 & Landsat (TM) & 30 \\
1995 & Landsat (TM) & 30 \\
2003 & QuickBird & 0.65 \\
2005 & Landsat (TM) & 30 \\
& QuickBird & 0.65 \\
2010 & Landsat (ETM) & 15 \\
& GeoEye-1 & 0.46 \\
2015 & Landsat (ETM) & 15 \\
& WorldView-2 & 0.46 \\
\hline
\end{tabular}

Table 1: Landsat Images Captured over 40-Year Period

\section{METHODOLOGY}

Figure 2 shows the framework of the proposed methodology. Prior to quantifying built-up areas in Landsat and VHSR images (QuickBird, GeoEye_1 and WorldView_2), image-image registration was conducted to ensure that conjugate features on the multi-temporal images belong to the same features on the ground (Boardman et al., 1996; Fonseca and Manjunath, 1996; Hsieh et al., 1997). The registered images therefore were used to quantify the built-up area representing urbanization using two approaches. The first approach relied on image processing techniques and accurate image registration procedures using multi-temporal Landsat satellite images. It utilized the derived edges (roads and buildings) from images as the basis of change detection. The second approach relied on digitized features to provide a ground truth for the edges (extracted from Landsat). The methodology utilized edge images that mitigate the sensitivity to the surface reflectance of bands of Landsat images and the effect of radiometric differences between the images in question.

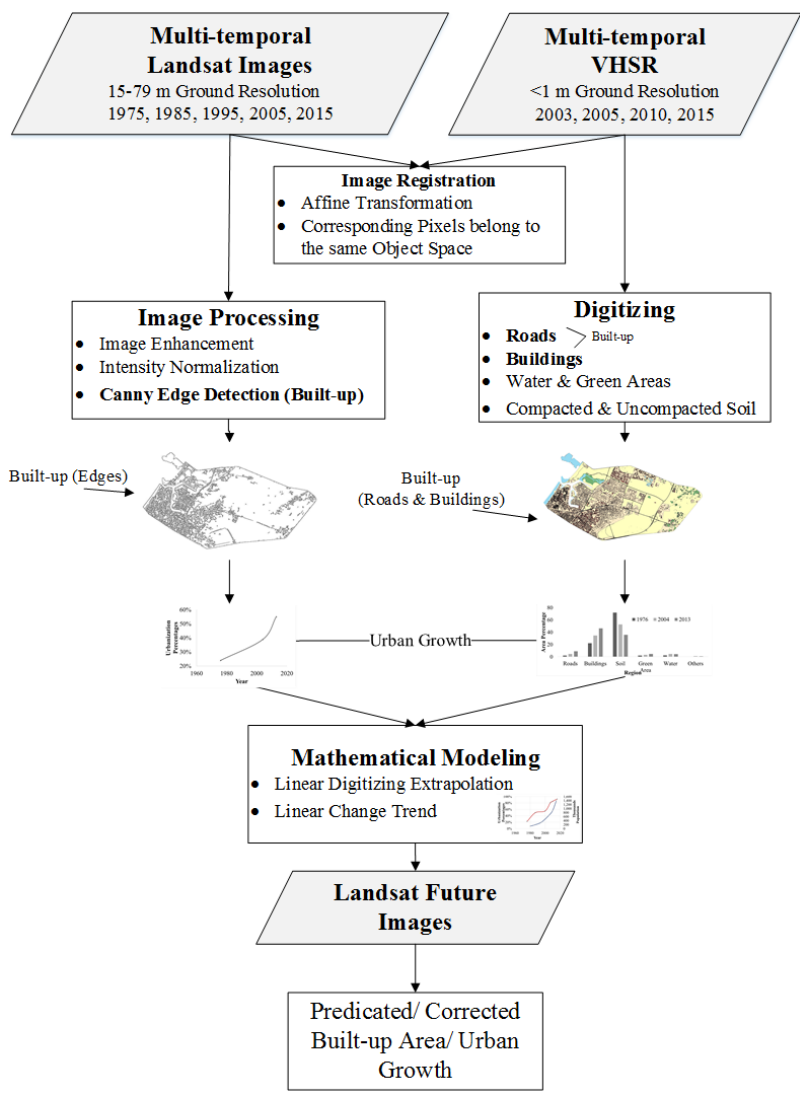

Figure 2. Framework of Proposed Methodology

\subsection{Image Registration}

Image registration aims at spatially aligning two images so that corresponding pixels in the image space belong to the same feature in the object space. In this work, image registration has been applied to the collected Landsat and VHSR images that have different spatial and temporal resolutions. The registration procedure also involves establishing a transformation function which mathematically describes the mapping between the imageries in question. In other words, the transformation function attempts to properly overlay the input images (Landsat) to fit the reference image (VHSR). Considering the fine topographic nature of the study area, Affine transformation was used as the registration transformation function. This is a valid function for imagery captured by satellite imaging systems with narrow angular field of view over relatively flat terrain as compared to the flying height (Al-ruzouq et al., 2011). Image registration is a prerequisite for reliable change detection, the accuracy of the registration process is the key factor that controls the validity and reliability of the change-detection outcome (Al-Ruzouq et al., 2017).

\subsection{Image Processing and Edge Extraction}

After finalizing image registration, where corresponding features in images refer to the same object space, intensity normalization was conducted to mitigate effects of radiometric differences and to ensure same intensity mean and variance values. Instead of working with the original images after intensity modification, derived edge images can be used as a basis for the change-detection procedure (Al-ruzouq et al., 2011). Using edge images has two advantages: first, extracted edges are robust to various radiometric differences between the 

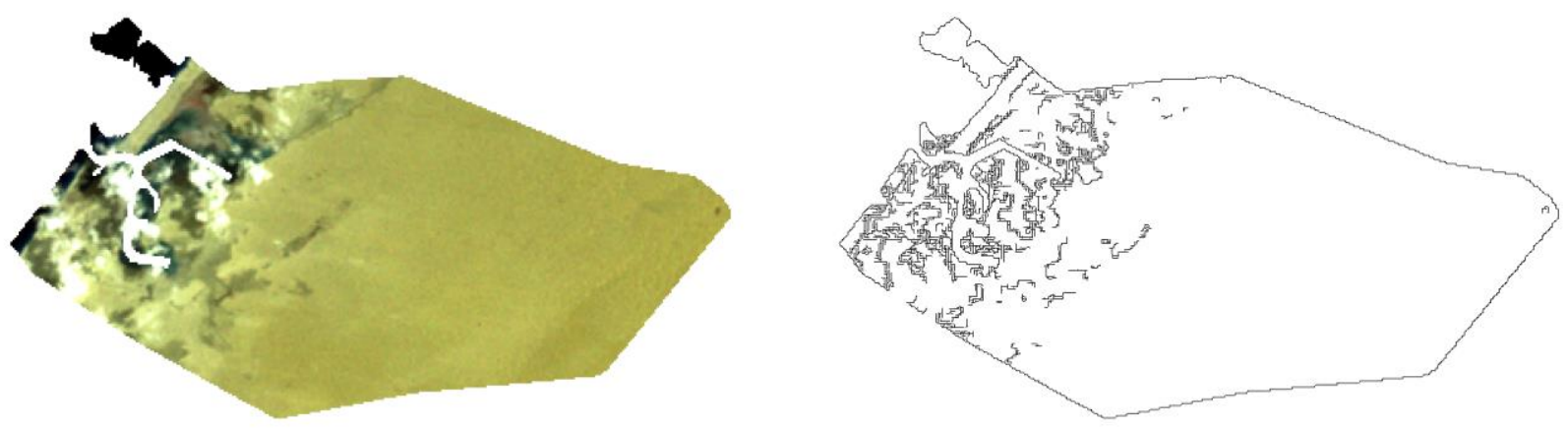

1975
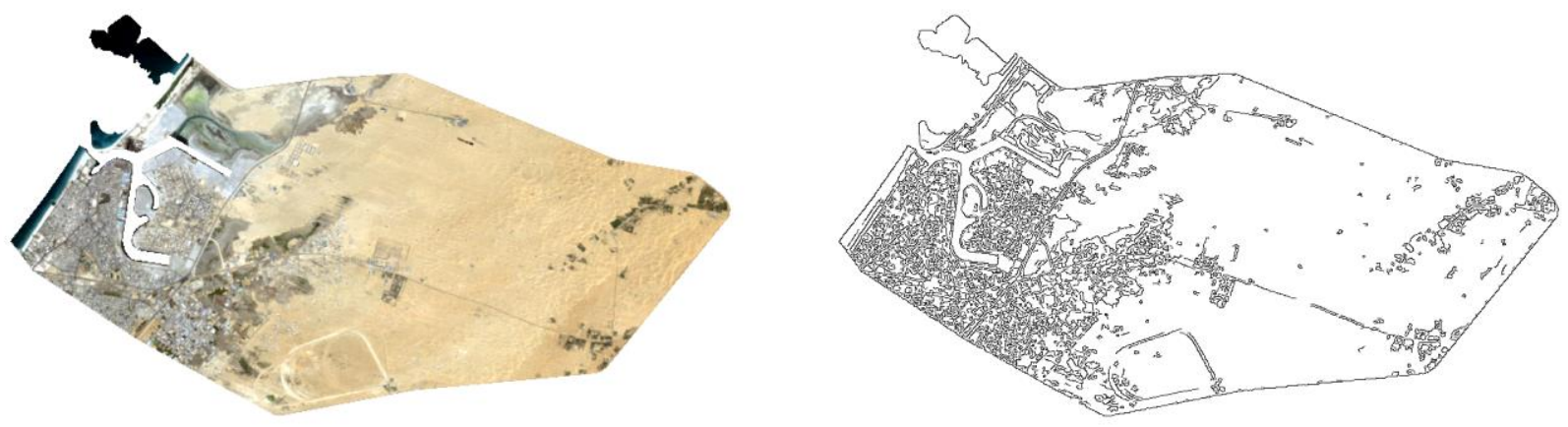

1995
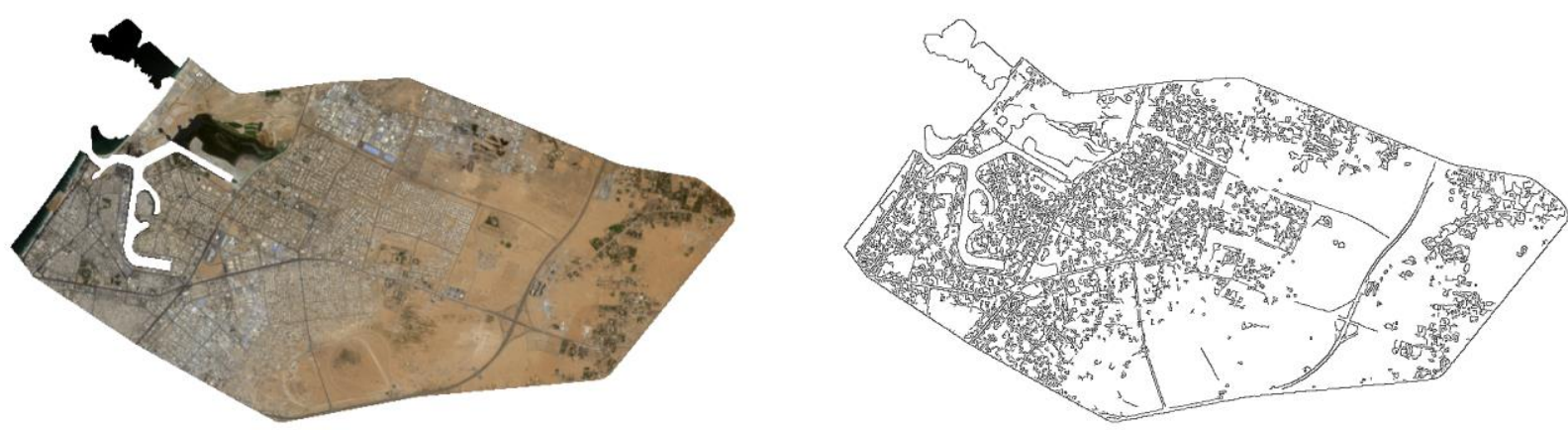

2015

(a)

(b)

Figure 3: (a) Multi-Temporal Landsat Images for Ajman City; (b) Edge images

registered images and second, the edges would represent urban features, such as roads and building boundaries.

In this study, the Canny edge detector (Canny, 1986) was used to extract edge pixels from Multi-temporal Landsat images. Canny Edge Detection utilize Gaussian filters that result in good detection, good localization, and a single response to an edge (Hashim et al., 2013). The algorithm uses Gaussian filter smooth and reduces the noise in the image. The pixels that have specific differences from the surrounded pixels (threshold) would be classified as edge pixels. Figure 3(b) shows the extracted edges for multi-temporal Landsat images.

\subsection{Spatial Analysis and Feature Digitizing}

The second choice for quantifying the built-up areas that representing urbanization relied on digitizing features from
VHSR images. In this process, a tracing in a geographically correct way to various spatial information from images/maps, such as buildings, roads, and land characteristics. The digitizing process intended mainly to provide a ground truth for the urban growth monitoring by the image processing mentioned in the previous section. Having map features in a digital format allows for various types of spatial analyses and interpretation. Examples of such analyses include calculating geometric properties of features and identifying topological relationships among features. As mentioned before, this process needs a hard work form a skilled operator. Figure 4 (c) shows Landover categories for the study area that include roads, building, green area, water, compacted and non-compacted soil. Figure 4(d) shows close up sample of the digitized features. 


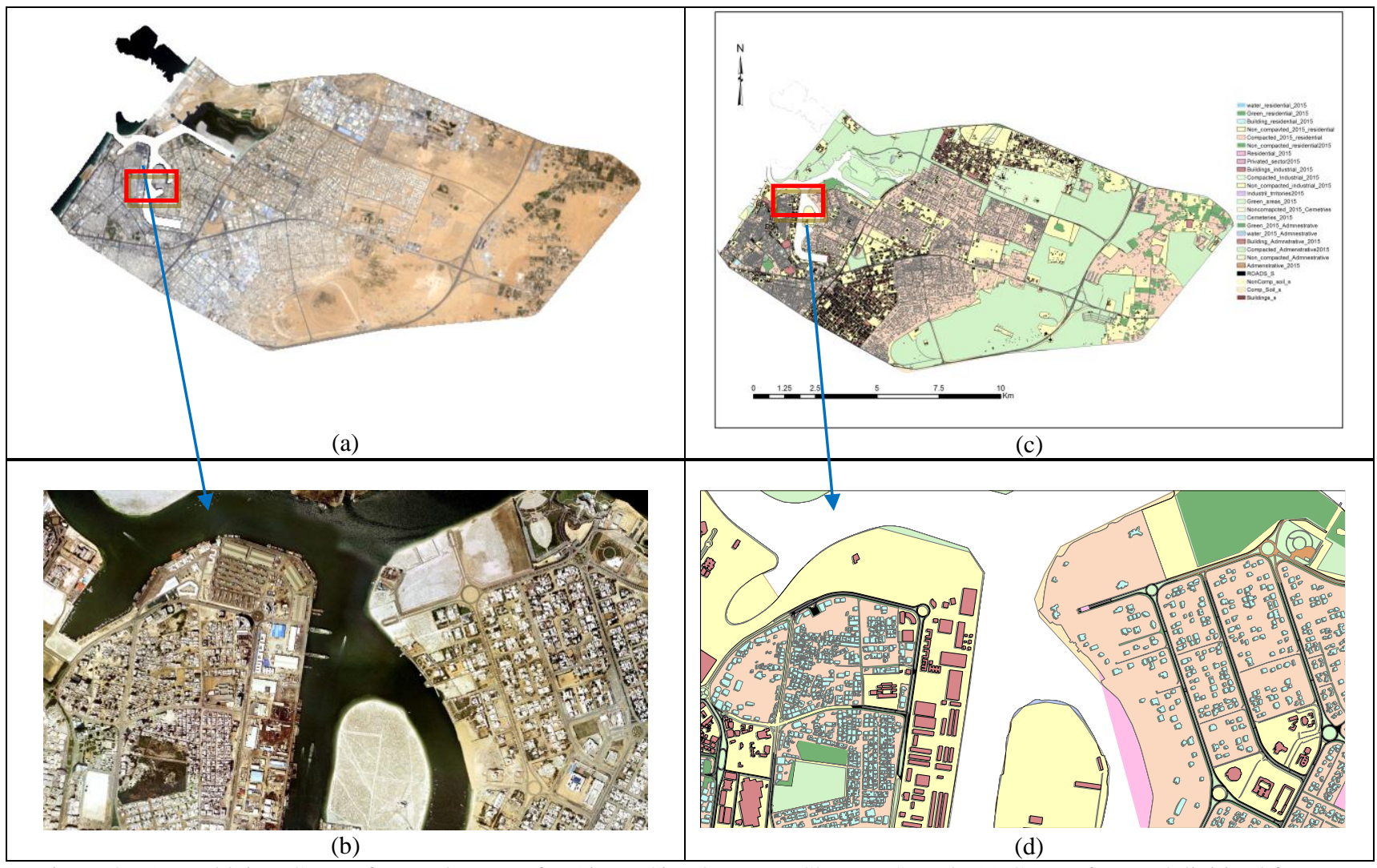

Figure 4: (a) Worldview Geo-Referenced Images for Ajman City (b) Image Close up Sample (c) Geo-Referenced digitized features for Ajman City (b) Digitized Close up Sample.

\section{RESULTS AND DISCUSSION}

This section presents the resulting graphs, quantification, and validation that took for this study over Ajman City, UAE. The section consists of four subsections; first subsection discusses the outcome of the image-processing technique utilizing canny edge detection, as well as the subjective validation of these results using visual detection of anomalies; the second subsection, the outcome of the digitizing analysis is presented and discussed thoroughly. It is worth reminding here that the results of the digitization were used as a ground truth to verify the outcome of the first approach; The third subsection shows the quantified pace of urbanization expansion that took place in Ajman City during the last four decades; last part discusses the mathematical calibration and modelling that relate the extracted edges to digitalized ground truth features.

\subsection{Edge Detection Results}

Based on the processed edge images, the area of edges for roads and buildings were calculated and are shown in Table 2.

\begin{tabular}{|c|c|c|}
\hline Year & \multicolumn{2}{|c|}{ Urban Area } \\
\hline & $\mathrm{Km}^{2}$ & \% with Respect to Total Area \\
1975 & 6.2 & 4.3 \\
1985 & 7.7 & 5.4 \\
1995 & 16.4 & 11.5 \\
2000 & 17.4 & 12.2 \\
2010 & 20.0 & 14.0 \\
2015 & 20.9 & 14.6 \\
\hline
\end{tabular}

Table 2: Landsat Images Captured over 40-Year Period

Figure 5 shows a graphical representation of the urbanization growth over 40 years.

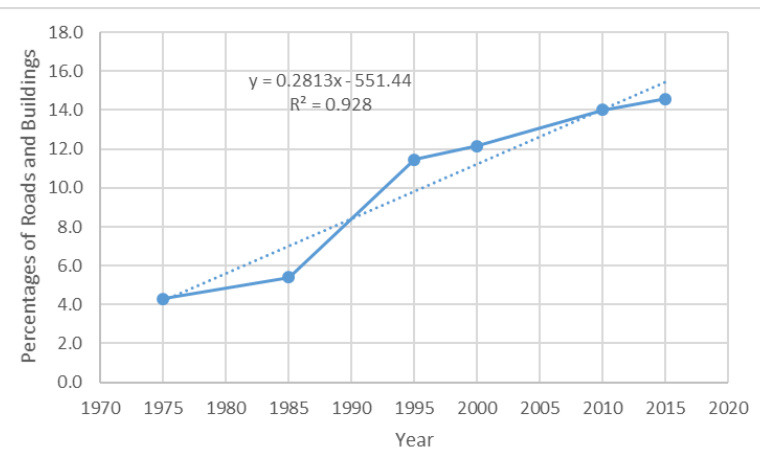

Figure 5. Urban growth from 1975 to 2016 based on edges

As shown in Figure 4, the urbanization trend in Ajman City can be visually observed. A regular expanding pattern off the coastal areas can be viewed over the 40-year period. This expansion extends from the south-eastern corner toward the central-south part of the city. Another observation is the concentration of the buildings and roads around the lagoon in the downtown area after 1995. Moreover, a remarkable change has occurred in the water body in the north-western part of the city, where some water bodies have been expanded as part of the city's plan to attract tourists and vacationers. An interesting part can be noticed after that the north-western part of the city does not host residential and commercial real estate as a result of establishing nature reserve, resort and recreation activities.

Figure 6(a) shows the limited roadway network in 1995 where Figure 6(b) shows the expansion of the roadway network in the same area that occurred by 2010 as part of infrastructure development. The change in buildings can also be clearly noticed at the center of the city. 


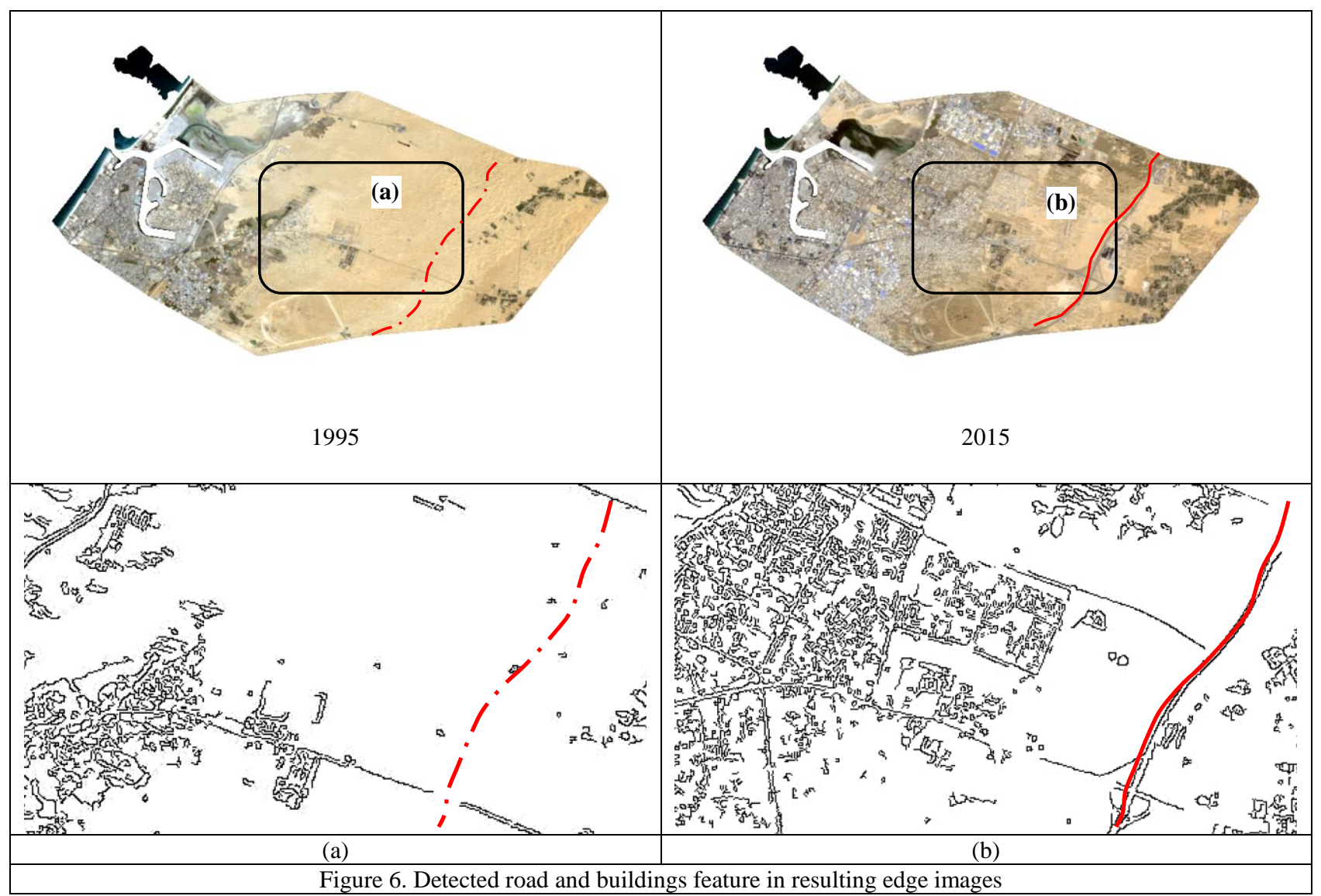

\subsection{Digitized Features Analysis}

Even though the focus of this paper is on urbanization (i.e., road and building features only), the digitized land cover/land use features were digitized and classified into six classes: roads, buildings, compact and uncompact soil, vegetation and other. Figure 7 shows the distribution of different classes along 40 years.

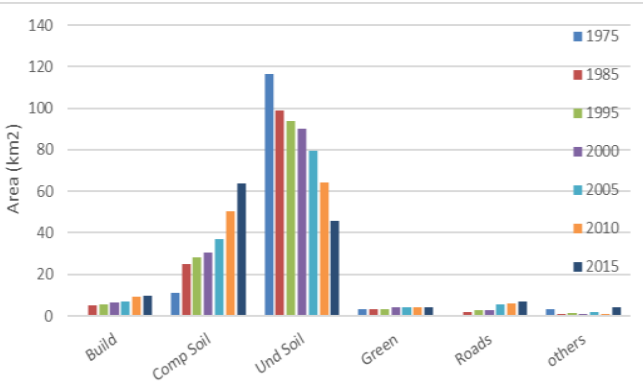

Figure 7. Distribution of Digitized Land Cover/Land Use

Features form 1975-2015. In particular, the built-up area (roads and building) are shown in Table 3 and Figure 8.

\begin{tabular}{|l|c|c|c|c|}
\hline \multirow{2}{*}{ Year } & \multicolumn{4}{|c|}{ Urban Area } \\
\cline { 2 - 5 } & & Roads & buildings & Total \\
\hline & $\mathrm{Km}^{2}$ & $\%$ & $\%$ & $\%$ \\
2000 & 10.8 & 2.9 & 4.6 & 7.5 \\
2005 & 11.6 & 2.8 & 5.3 & 8.1 \\
2010 & 15.9 & 3.1 & 8.1 & 11.1 \\
2015 & 17.9 & 3.9 & 8.6 & 12.5 \\
\hline
\end{tabular}

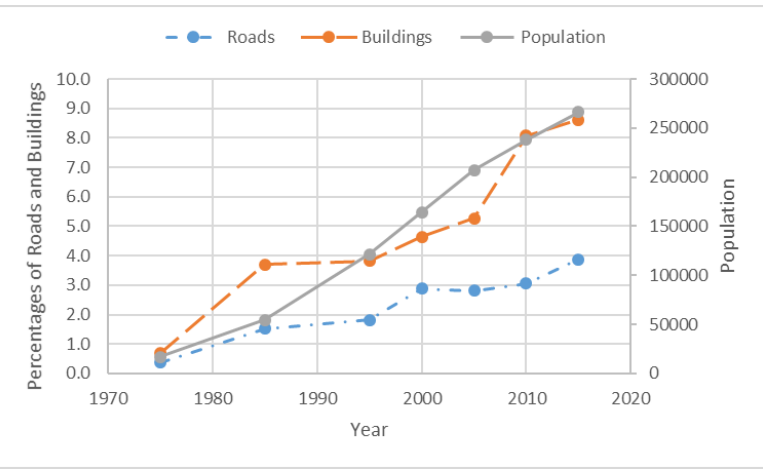

Figure 8. Distribution of Digitized Land Cover/Land Use

While roads formed about $0.4 \%$ of the total area of the city in 1975, they accounted around than $4 \%$ in 2015. Similarly, buildings accounted for nearly $0.7 \%$ in 1975 but more than 12 fold to reach about $8.6 \%$ in 2015 . It can be noted that roads and building development did grow almost parallel. Figure 8 also shows the dramatic growth trends of population reflect the rapid economic growth of Ajman and the UAE. The observed decrease of the development rate of buildings compared to population due to the fact that Ajman City witnessed a horizontal expansion of buildings in the first three decades but more of vertical expansion of buildings in the last decade. 


\subsection{Calibration Prediction and Validation}

In summary, two approaches complemented each other in demonstrating urbanization patterns in Ajman City. The first approach, which depended on image processing, was easy to implement with minimum human effort. It was less sensitive to utilizing images with various spatial resolutions. The second approach, which depended on digitized features represent the ground truth. Its major disadvantages are that it is prone to human error and it is resource-consuming.

Two methods were used to predict the future growth considering absence and high cost of VHSR images as well as escaping the hard digitizing process; first method simply relies on linear regression to model the relationship between time and digitized built up area. Figure 9 shows the linear relation based with the 5-year period step from 1995 to 2010. The year of 2015 was not involved and reserved for validation. Applying the linear regression, to predict the built up in 2015, result in less than $1 \%$ error. While archived digitized built-up area is not always available, relying on medium resolution, such as Landsat, and above-mentioned edge extraction would be valuable and significant.

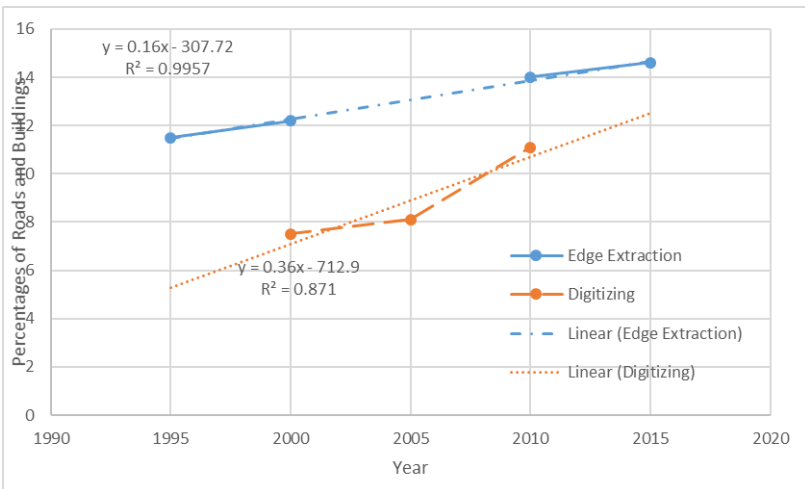

Figure 9. Linear regression modeling based on edge extraction and digitizing

Therefore, the adopted second method to predict the built-up area in this study depends on developing linear regression between time and the difference in built-up area between digitized features and extracted edges. Table 4 contains the difference in percentage in built-up area based on edge extraction and digitizing. Note that years 1975 and 1885 were not used in the edge prediction model due to the expected low of accuracy associated with their course resolution. It is also important to mention that the built-up area appears in Table 4 (edge extraction of year 2005, and digitizing of year 1995) were extracted using previous developed linear regressions.

\begin{tabular}{|c|c|c|c|}
\hline Year & Edge Extraction & Digitizing & difference \\
\hline & $\%$ & $\%$ & $\%$ \\
1995 & 11.5 & 5.3 & 6.2 \\
2000 & 12.2 & 7.5 & 4.7 \\
2005 & 13.1 & 8.1 & 5.0 \\
2010 & 14.0 & 11.1 & 2.9 \\
\hline
\end{tabular}

Table 4: Edge Extraction -Digitizing percentage difference in Built up area based on two approaches; edge extraction and digitizing.
Figure 10 demonstrates the for Linea trend $r$ that represent the difference in built-up area resulted from both edge extraction and digitizing.

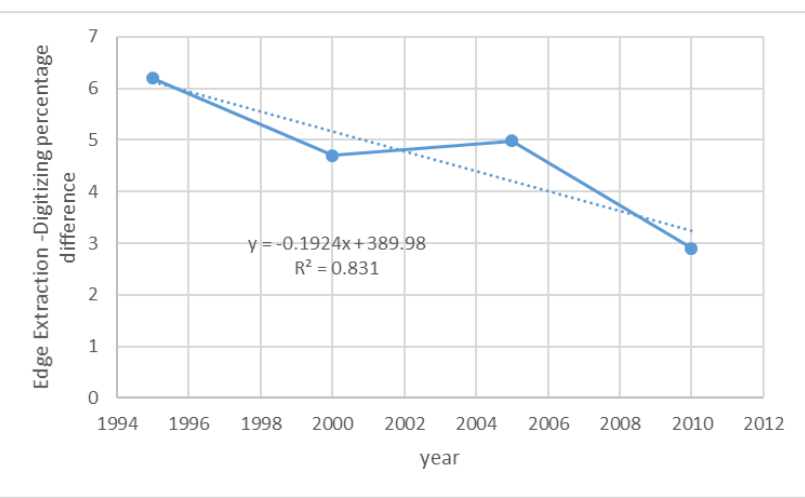

Figure 10. Linear regression modeling edge extraction digitizing difference percentage

The differences model has been applied to predict the defiance in 2015. Afterword, the difference value was subtracted from the built-up area (detecting edges 2015) to obtain the predicted built up area $1 \mathrm{n} 2015$. To validate the result, the percentage error was calculated based on actual digitized built up area AND found to be $6.4 \%$.

\section{CONCLUSION AND FUTURE WORK}

This paper presented two methods for quantifying and predicting urban growth. Multi-temporal satellite images with various spatial and radiometric resolutions have been used. The first approach relies on available low-cost medium resolution to detected edges (roads and buildings) using the Canny Edge Detection operator while the second approach directly utilized digitized features VHSR images. Two prediction models have been developed; the first depends on linear regressing of temporal digitized images and second approach depend on linear regression of differences between extracted and digitized features. Even first prediction model reveal lower error than difference model. The extracted edges are quite important considering cost, availability and processing efforts to satellite images.

As a case study, the proposed methodology was applied to detect urbanization growth in Ajman City, UAE. To apply the first approach, the authors utilized Landsat images from 1975 to 2015 while VHSR images were utilized for the second approach. The results of both approaches shown that Ajman City has experienced dramatic urbanization expansion comparable to its population growth over the past 40 years. While urbanized areas constituted slightly less than $1 \%$ of the total area in 1975, built up has considerably expanded to cover roughly $12.5 \%$ in 2015

The process of urbanization has a considerable hydrological impact in terms of influencing the nature of runoff. future work will be dedicated to examine the effect of such urban growth on surface runoff and evaluating the impact of urban growth on rainfall-Runoff relationship.

\section{ACKNOWLEDGEMENTS}

We would like to acknowledge Ajman Municipality for the continues support provided along this study. 


\section{REFERENCES}

Al-Ruzouq, R., Hamad, K., Shanableh, A., Khalil, M., 2017. Infrastructure growth assessment of urban areas based on multitemporal satellite images and linear features. Ann. GIS 1-19. https://doi.org/10.1080/19475683.2017.1325935

Al-Ruzouq, R.I., Al-zoubi, A., Akawi, E.E., 2011. International Journal of Remote Multiple source imagery and linear features for detection of urban expansion in Aqaba City, Jordan 37-41. https://doi.org/10.1080/01431161.2011.616917

Aljoufie, M., Zuidgeest, M., Brussel, M., van Maarseveen, M., 2013. Spatial-temporal analysis of urban growth and transportation in Jeddah City, Saudi Arabia. Cities 31, 57-68. https://doi.org/10.1016/j.cities.2012.04.008

Boardman, D., Dowman, I., Chamberlain, A., Fritsch, D., Newton, W., 1996. An automated image registration system for SPOT data. Int. Arch. Photogramm. Remote Sens. 31, 128-133.

Bouziani, M., Goïta, K., He, D.-C., 2010. Automatic change detection of buildings in urban environment from very high spatial resolution images using existing geodatabase and prior knowledge. ISPRS J. Photogramm. Remote Sens. 65, 143-153. https://doi.org/10.1016/j.isprsjprs.2009.10.002

Elmahdy, S.I., Mohamed, M.M., 2016. Land use / land cover change impact on groundwater quantity and quality: a case study of Ajman Emirate, the United Arab Emirates , using remote sensing and GIS. Arab. J. Geosci. https://doi.org/10.1007/s12517-016-2725-y

Fonseca, L.M.G., Manjunath, B.S., 1996. Registration Techniques for Multisensor Remotely Sensed Imagery. Photogramm. Eng. Remote Sens. 62, 1049-1056.

Hsieh, J., Liao, H., Fan, K., Ko, M., Hung, Y., 1997. Image Registration Using a New Edge-Based Approach. Comput. Vis. Image Underst. 67, 112-130.

Jin, H., Mountrakis, G., 2013. Integration of urban growth modelling products with image-based urban change analysis. Int. J. Remote Sens. 34, 5468-5486. https://doi.org/10.1080/01431161.2013.791760

Li, Y., Wang, C., Li, Y., Wang, C., 2016. Impacts of Urbanization on Surface Runoff of the Dardenne Creek Watershed , St . Charles County , Missouri 3646. https://doi.org/10.2747/0272-3646.30.6.556

Liu, S., Member, S., Bruzzone, L., Bovolo, F., Member, S., 2015. Hierarchical Unsupervised Change Detection in Multitemporal Hyperspectral Images 53, 244-260.

Rimal, B., n.d. Monitoring and Modeling of Spatiotemporal Urban Expansion and Land-Use / Land-Cover Change Using Integrated Markov Chain Cellular Automata Model. https://doi.org/10.3390/ijgi6090288

Singh, A., 1989. Review Articlel: Digital change detection techniques using remotely-sensed data. Int. J. Remote Sens. 10, 989-1003. https://doi.org/10.1080/01431168908903939

Song, X., Sexton, J.O., Huang, C., Channan, S., Townshend, J.R., 2016. Remote Sensing of Environment Characterizing the magnitude, timing and duration of urban growth from time series of Landsat-based estimates of impervious cover. Remote Sens. Environ. 175, 1-13. https://doi.org/10.1016/j.rse.2015.12.027
Suriya, S., Mudgal, B. V., 2012. Impact of urbanization on flooding: The Thirusoolam sub watershed - A case study. J. Hydrol. 412-413, 210-219. https://doi.org/10.1016/j.jhydrol.2011.05.008

Tanr, H., Canaz, S., 2017. Change detection using Landsat images and an analysis of the linkages between the change and property tax values in the Istanbul Province of Turkey 200, 446-455. https://doi.org/10.1016/j.jenvman.2017.06.008

Verma, R., Kumari, K., Tiwary, R., 2009. Application of Remote Sensing and GIS technique for efficient Urban planning in India. Geomatrix Conf. Proc. 1-23.

Wang, K., Zhou, W., Xu, K., Liang, H., Yu, W., Li, W., 2017. Quantifying Changes of Villages in the Urbanizing Beijing Metropolitan Region: Integrating Remote Sensing and GIS Analysis. https://doi.org/10.3390/rs9050448

Yildiz, S., Doker, M.F., 2016. Monitoring urban growth by using segmentation-classification of multispectral Landsat images in Izmit, Turkey. https://doi.org/10.1007/s10661-016$5392-2$

Zope, P.E., Eldho, T.I., Jothiprakash, V., 2016. Catena Impacts of land use - land cover change and urbanization on fl ooding : A case study of Oshiwara River Basin in Mumbai , India. Catena 145, 142-154. https://doi.org/10.1016/j.catena.2016.06.009 